\title{
Figures of Merit for CMOS Low-Noise Amplifiers and Estimates for Their Theoretical Limits
}

\author{
Leonid Belostotski, Senior Member, IEEE, and Eric A.M. Klumperink, Fellow, IEEE
}

\begin{abstract}
This paper examines the theoretical interdependence of lownoise-amplifier (LNA) performance parameters to identify four figures of merit (FoMs) that enable the comparison of LNAs. FoM limits are also estimated to identify performance limitations and to quantify room for theoretical improvement. Finally, the results of a regression analysis on nearly 500 CMOS LNAs, published over the past 20 years, are compared to the identified trends, with the findings showing reasonable agreement despite the adoption of some simplifying assumptions.
\end{abstract}

Index Terms-CMOS, figure of merit, FoM, LNA, linearity measure

\section{INTRODUCTION}

$\mathbf{N}$ EW CIRCUITS are constantly being developed for commercial products and research projects. These new circuits feature diverse sets of specifications and often provide novel solutions to the limitations of prior circuits.

Circuit surveys [1]-[6] and performance benchmarking using figures of merit (FoMs) [7], [8] can help to find viable circuit solutions and assess achievable performance. While numerous FoMs have been proposed, their relevance to key design trade-offs is not always obvious (over 30 different FoMs were identified during the compilation of the survey [6]!).

If an FoM captures a key design trade-off, one would expect this to be corroborated by practical data points. To this end, a regression analysis was applied on the data in [6] to identify the underlying trade-offs for low-noise amplifiers (LNAs) [9]. Such regression analysis treats all data points as equally relevant, i.e. it focuses on "typical" rather than cutting-edge performance. Although the regression-based FoMs in [9] identify trade-offs buried in the data, the resultant FoMs are not static, as they are adjusted every time new data is added to the survey. In addition, this type of analysis does not identify theoretical limits.

The contribution and objective of this article is to identify theoretical trade-offs that can be captured in a model to serve as a basis for LNA-FoMs and for estimating their theoretical limits. Such FoMs can serve as benchmarks to guide designers with respect to what is theoretically possible. Although these theoretical limits may model best-case scenarios that are virtually unreachable in practice, they are nevertheless useful for at least two reasons: 1) they quantify how much theoretical room is available for performance improvement; and 2) they help to identify factors that are limiting the potential of practical designs, which might trigger ideas for improving the state of the art. We assume that such FoMs should be based on commonly published data.

While the quality of power and noise matching are not typically included in FoMs, such matching is often important and constrains LNA design. Section II begins by reviewing the minimum noise factor $\left(F_{m i n}\right)$ and the optimum admittance $\left(Y_{o p t}\right)$ required to reach $F_{m i n}$, as well as the condition required for simultaneous noise and

Manuscript received on XXXX XX, 202X. This work was supported by The University of Calgary, the Natural Sciences and Engineering Research Council of Canada, Canada Reasearch Chair Program, and CMC Microsystems.

L. Belostotski is with the Department of Electrical and Software Engineering, University of Calgary (e-mail: lbelosto@ucalgary.ca). E.A.M. Klumpernik is with the Faculty of Electrical Engineering, Mathematics and Computer Science, University of Twente (e-mail: e.a.m.klumperink@utwente.nl). power matching. We propose employing a classical multi-metrics noise measure and a linearity measure to capture system-performance trade-offs that constrain LNA designs. In Section III, equations for estimating theoretically achievable performance are derived, and the trade-offs with operating frequency, technology node, noise, and power consumption are identified. To keep analysis tractable, seven assumptions are made, justified, and stated explicitly to clearly identify the theoretical boundary conditions for the claims made and the resultant best achievable performance identified. Section IV presents the proposed FoMs and their limits, while Section V discusses the similarity between proposed FoMs and the FoMs obtained via a regression analysis on a dataset of more than 500 LNAs.

\section{LNA Performance Metrics: Minimum Noise Factor, NOISE AND LINEARITYMEASURES}

Common LNA performance metrics include operating frequency $\left(\omega_{0}=2 \pi f_{0}\right)$, spot noise figure/factor $(F)$ in a $1-\mathrm{Hz}$ bandwidth, gain $(G)$, bandwidth $(\Delta f), 1-\mathrm{dB}$ compression point $\left(\mathrm{P}_{1 \mathrm{~dB}}\right)$, 3rd-order intercept (IP3), and power consumption $\left(P_{d}\right)$. On their own, these metrics are valid for comparing LNAs; however, they do not reflect design trade-offs and system-performance trade-offs. As the result this section reviews the fundamental $F_{\min }$ and noise measure and proposes a linearity measure to serve as key FoM components.

\section{A. Minimum Noise Factor $\left(F_{\min }\right)$}

$F_{\min }$ represents the fundamental limit on the best possible noise factor from a transistor. For a MOSFET [10], [11]

$$
F_{\text {min }}=1+2 \frac{\omega_{0}}{\omega_{T}} \sqrt{\frac{\delta \gamma}{5}\left(1-|c|^{2}\right)}
$$

where $\delta$ and $\gamma$ are the excess noise coefficients for the induced gate and drain noise currents, $c$ is the correlation coefficient between these noise currents, and $\omega_{T}$ is the frequency of the unity current gain. $F_{\min }$ is achieved, when the signal-source admittance equals the optimum admittance for minimum noise, $Y_{o p t}=G_{o p t}+j B_{o p t}$, where [10], [12]-[15]

$$
\left\{\begin{array}{l}
G_{o p t}=\omega_{0} C_{g s} \alpha \sqrt{\left(1-|c|^{2}\right) \delta / 5 \gamma} \\
B_{o p t}=-\omega_{0} C_{g s}(1-\alpha|c| \sqrt{\delta / 5 \gamma}) .
\end{array}\right.
$$

In (2), $C_{g s}$ is the gate-source capacitance and [16]

$$
\alpha=\frac{1+0.5 \rho}{(1+\rho)^{2}} \text { with } \rho \equiv \frac{V_{\text {od }}}{L E_{\text {sat }}},
$$

where $V_{o d}$ is the overdrive voltage, $L$ is the channel length, and $E_{\text {sat }}$ is the velocity-saturation field strength [10], [17].

Ideally, an LNA design achieves simultaneous noise and power matching, which requires $Y_{i n}=Y_{o p t}^{*}$. Many prior publications have noted that such simultaneous matching is not feasible as $B_{\text {opt }} \neq \Im\left\{Y_{i n}\right\}$, where $\Im\left\{Y_{i n}^{*}\right\}=\omega C_{g s}$. However, prior works have simplified the mean-squared drain and gate noise current expressions, $\overline{i_{d n}^{2}}=4 k_{b} T_{0} B \gamma g_{d o}$ and $\overline{i_{g n}^{2}}=4 k_{b} T_{0} B \delta \omega_{0}^{2} C_{g s}^{2} / 5 g_{d 0}$, by replacing $g_{d 0}$ (i.e., the output conductance when $V_{d s}=0$ ) with transconductance $g_{m}$ rather than $g_{m} / \alpha$, where $k_{b}$ is Boltzmann's 
constant, $T_{0}$ is the reference temperature, and $B$ is the noise bandwidth. Recent work [18] exploits parameter $\alpha$ for power and noise matching. The optimum $C_{g s}$ was derived, which served to minimize $\Delta F=F-F_{\min }$ and reduce the sensitivity, $S_{f} \equiv \frac{\partial \Delta F}{\partial \omega} \frac{\omega}{\Delta F}$, of $\Delta F$ to $\omega$. It was found that

$$
\alpha_{o p t}=|c| \sqrt{\frac{5 \gamma}{\delta}}
$$

is optimum as it makes $\Im\left\{1 / Y_{\text {opt }}\right\}=\Im\left\{1 / Y_{i n}^{*}\right\}=1 / \omega_{0} C_{g s}$ as desirable for simultaneous noise and power matching. E.g., inductive source degeneration realizes $\Re\left\{1 / Y_{\text {opt }}\right\}=\Re\left\{1 / Y_{i n}^{*}\right\}$ while preserving $\Im\left\{1 / Y_{o p t}\right\}=\Im\left\{1 / Y_{i n}^{*}\right\}$. This analysis has two consequences for this article:

- it gives equations for $\alpha_{o p t}$ and $\rho_{\text {opt }}$ (via (3)) that provide simultaneous noise and power matching,

- for the signal-source impedance, $Z_{0} \in \mathbb{R}$, both $\Delta F$ and its sensitivity to $\omega$ are minimized when

$$
\omega_{0} C_{g s}^{o p t}=\frac{1}{Z_{0}} \frac{|c|}{\sqrt{1-|c|^{2}}} .
$$

\section{B. Noise Measure}

Since LNAs are intended to reduce system noise factor, LNA $F$ should always be in an LNA FoM. Furthermore, gain $(G)$ is also intuitively important for LNAs. How to account for this trade-off is a research question that dates back to Haus and Adler's [19] work on defining "noise measure." This stepping-stone to defining an FoM was derived by starting with Friis' formula [20]

$$
F_{12}=F_{1}+\frac{F_{2}-1}{G_{1}} \text { and } F_{21}=F_{2}+\frac{F_{1}-1}{G_{2}},
$$

where $F_{12(21)}$ and $F_{1(2)}$ are the noise factors of the cascade of LNA \#1-\#2 (LNA \#2-\#1) and LNAs \#1(\#2), respectively, and $G_{1(2)}\left[\right.$ lin] are their available gains. For $F_{12}<F_{21}$, (6) results in

$$
\frac{F_{1}-1}{1-G_{1}^{-1}}<\frac{F_{2}-1}{1-G_{2}^{-1}}
$$

which shows that the lowest noise factor for a cascade is achieved by positioning the LNA with the lowest

$$
F_{M} \equiv \frac{F-1}{1-G^{-1}},
$$

rather than the lowest $F$, at the front. $F_{M}$ is the noise measure.

Some important conclusions stemming from [19] include:

1) The minimum of $F_{M}$ is device-size invariant.

2) $F_{M}$ of an arbitrary number of two-port amplifier stages with lossless interconnections is not less than the best-stage $F_{M}$.

3) $F$ of a network cannot be less than $F$ of the best stage.

4) Lossy or active-circuit embedding adds noise and can only degrade the minimum $F_{M}$.

While the noise measure connects the gain and noise factor, it does not link power consumption and other performance metrics to LNA noise. As [19] demonstrates, the best possible performance in terms of noise can only be achieved with a single-transistor, which is hence the focus of Section III.

\section{Linearity Measure}

Analogously to noise measure, in this paper linearity measure is derived from the input-referred linearity of a two-LNA cascade [10]

$$
\frac{1}{I P_{12}} \approx \frac{1}{I P_{1}}+\frac{G_{1}}{I P_{2}} \text { and } \frac{1}{I P_{21}} \approx \frac{1}{I P_{2}}+\frac{G_{2}}{I P_{1}}
$$

where $I P_{12(21)}[\mathrm{mW}]$ is the input-referred $\mathrm{P}_{1 \mathrm{~dB}}\left(\mathrm{IP}_{1 \mathrm{~dB}}\right)$ or IP3 (IIP3) of the cascade of LNA \#1(\#2) and LNA \#2(\#1), and $I P_{1(2)}[\mathrm{mW}]$ and $G_{1(2)}$ [lin] are the $\mathrm{IP}_{1 \mathrm{~dB}}$ or IIP3 and gains of LNAs \#1(\#2). If $P_{1 d B, 12}>P_{1 d B, 21}$, then (9) gives

$$
I P_{1}\left(G_{1}-1\right)<I P_{2}\left(G_{2}-1\right) .
$$

Based on (10), the $\mathrm{IP}_{1 \mathrm{~dB}}$ linearity measure can be defined as

$$
P_{L} \equiv I P_{1 d B}(G-1) .
$$

As shown in (10), the $\mathrm{IP}_{1 \mathrm{~dB}}$ of a cascade can be maximized by placing the amplifier with the larger $P_{L}$ last. $P_{L}$ captures the trade-off between gain and linearity, which is useful in defining FoMs.

\section{KEY EQUATIONS}

This section builds on Section II and investigates the theoretical properties of single-transistor LNAs in order to identify FoMs and their theoretical limits. To provide insight and keep the math tractable, simple modeling is used based on the following assumptions:

Assumption \#1: Transistors biased in strong inversion and saturation are assumed, as their $F_{\min } \mathrm{s}$ are lower than in subthreshold.

Assumption \#2: The small-signal behavior of a MOSFET in saturation is modeled with only $g_{m}$ and $C_{g s}$.

Assumption \#3: Simultaneous noise and power matching as described in Section II-A is targeted. ${ }^{1}$

Assumption \#4: A simple second-order model of the MOSFET current is employed [16]

$$
I_{d}=C_{o x} W v_{s a t} \frac{V_{o d}^{2}}{V_{o d}+L E_{s a t}},
$$

where $V_{o d}=V_{g s}-V_{T}, V_{g s}$ is the gate-source voltage, $V_{T}$ is the threshold voltage, $L$ and $W$ are the channel length and width, $C_{o x}$ is the gate oxide capacitance per area, and $v_{\text {sat }}$ is the saturation velocity.

Assumption \#5: $V_{d d}=V_{d s}=V_{o d}$ is assumed for minimum power consumption, $P_{d}$, while avoiding triode. Using (3), (12) gives

$$
P_{d}=V_{d d} I_{d}=V_{o d} I_{d}=\frac{C_{o x} W \mu_{e f f}}{2 L(1+\rho)} V_{o d}^{3},
$$

where $\mu_{e f f} \equiv 2 v_{\text {sat }} / E_{\text {sat }}$ is the field-limited charge mobility.

Assumption \#6: Long-channel parameters $\gamma=2 / 3, \delta=4 / 3$, and $c=j 0.395$ are assumed. Assumptions \#3 and \#6 and (4) give $\alpha_{o p t}=$ 0.62 and $\rho_{\text {opt }}=0.4$ via (3).

Assumption \#7: For determining linearity-based FoMs, it is assumed that linearity can be assessed via (12).

With these assumptions, the key expressions can be derived.

Overdrive voltage $\left(V_{o d}\right)$ : From (12) in Assumption \#4

$$
V_{o d}=\sqrt{\frac{2 L}{(1+\rho) C_{o x} \mu_{e f f}} \times \frac{I_{d}}{W}}
$$

Together with (13) from Assumption \#5, (14) expresses the relationship between $I_{d} / W$ and power consumption as follows

$$
\frac{I_{d}}{W}=\sqrt[3]{\frac{P_{d}^{2} C_{o x} \mu_{e f f}(1+\rho)^{5}}{2 L W^{2}}} .
$$

Transconductance $\left(g_{m}\right)$ : The differentiation of (12) with respect to $V_{g s}$ results in

$$
g_{m}=C_{o x} W \mu_{e f f} \frac{V_{o d}}{L} \frac{1+0.5 \rho}{(1+\rho)^{2}}=C_{o x} W \mu_{e f f} \frac{V_{o d}}{L} \alpha .
$$

${ }^{1}$ While methods, such as inductive source degeneration, may be required to complete matching, they do not affect equations in this paper. 
Using (14) and (15), (16) becomes

$$
g_{m}=\alpha W \sqrt{\frac{2 C_{o x} \mu_{e f f}}{L}} \sqrt[6]{\frac{P_{d}^{2} C_{o x} \mu_{e f f}(1+\rho)^{2}}{2 L W^{2}}}
$$

and relates $g_{m}$ to power consumption and transistor width.

Frequency of unity current gain $\left(\omega_{T}\right)$ : Combining (17), $C_{g s}=$ $2 / 3 C_{o x} W L$, and $\omega_{T}=g_{m} / C_{g s}$ (Assumption \#2) gives

$$
\omega_{T}=\frac{3}{2} \alpha \sqrt{\frac{2 \mu_{e f f}}{C_{o x} L^{3}}} \sqrt[6]{\frac{P_{d}^{2} C_{o x} \mu_{e f f}(1+\rho)^{2}}{2 L W^{2}}} .
$$

Transistor width $(W)$ : For a single MOSFET, the relationship between the width, $W$, and $C_{g s}=C_{g s}^{o p t}$ as per Assumption \#3 is obtained from (5) as

$$
W=\frac{3}{2 \omega_{0} C_{o x} L Z_{0}} \times \frac{|c|}{\sqrt{1-|c|^{2}}} .
$$

Minimum noise factor $\left(F_{\min }\right)$ : Substituting (18) and (19) into (1) and collecting all constants in to a constant $\mathcal{N}$ yields

$$
\frac{\left(F_{\text {min }}-1\right) P_{d}^{1 / 3}}{f_{0}^{2 / 3} L^{4 / 3}}=\frac{4}{\alpha} \sqrt{\frac{\delta \gamma}{5}} \sqrt[3]{\frac{\pi^{2}|c|\left(1-|c|^{2}\right)}{9 Z_{0} \mu_{e f f}^{2}(1+\rho)}}=\mathcal{N} .
$$

Linearity: The linearity of LNAs and other transconductancebased circuits is commonly characterized in terms of their IP3 and $\mathrm{P}_{1 \mathrm{~dB}}$ [21], [22]. While for a simple memoryless 3rd-order nonlinearity these values are often simply related as IP3 $=\mathrm{P}_{1 \mathrm{~dB}}+10 \mathrm{~dB}$ [21], [23], it is more complicated in general [22]. It is well-known that measured IP3 can be input-power dependent because for some power levels two nonlinearity effects may cancel each other out (e.g., due to derivative superposition) [24]. The tone spacing in two-tone IP3 measurements can also be responsible for modifying IP3 measurement results, but such tone spacings are not universally set equal. The addition of an output filter together with nonlinearity cancellation can reduce intermodulation products, thereby allowing IP3 to become very high, and almost unlimited. In contrast, the measurement procedure for $\mathrm{P}_{1 \mathrm{~dB}}$ is universal. Thus, $P_{L}\left(\mathrm{P}_{1 \mathrm{~dB}}\right)$ is selected for LNA FoMs in this work.

Feedback or gain expansion stages can boost $\mathrm{P}_{1 \mathrm{~dB}}$, potentially outperforming a stand-alone MOSFET. Therefore, expressions connecting $\mathrm{P}_{1 \mathrm{~dB}}$ to other LNA performance metrics can be highly specific to a sub-class of circuits rather than generally valid.

The data analysis in [6] shows that $\mathrm{P}_{1 \mathrm{~dB}}$ and $V_{d d}$ exhibit a $41 \%$ correlation and $\mathrm{P}_{1 \mathrm{~dB}}$ and $G$ exhibit a $12 \%$ correlation whereas $\mathrm{P}_{1 \mathrm{~dB}}$ and $P_{d}$ exhibit a $62 \%$ correlation and $P_{d}$ and $V_{d d}$ exhibit a $32 \%$ correlation. Since $\mathrm{P}_{1 \mathrm{~dB}}$ depends stronger on $P_{d}$ than $V_{d d}$, the onset of $\mathrm{P}_{1 \mathrm{~dB}}$ is often due to MOSFET nonlinear behavior rather than clipping, thus giving rise to Assumption \#7.

Using (16) and Assumption \#7, the condition for gain compression is investigated through the compression of $g_{m}$. For a given compression level $\left(G_{c}\right)$ and the input signal, $v_{s i g}=A \cos \left(\omega_{0} t\right)$, gain compression can be related to the average of $g_{m}$ via $\left\langle g_{m}\left(v_{i n}\right)\right\rangle=$ $\left.G_{c} g_{m}\right|_{v_{i n}=0}$, which, with the help of (16), becomes

$$
\begin{aligned}
\left.G_{c} g_{m}\right|_{v_{i n}}=0 & =\frac{C_{o x} W \mu_{e f f}}{2 A} \\
& \times \int_{-A}^{+A} \frac{\left(V_{o d}+v_{i n}\right)}{L} \frac{\frac{1}{2}\left(V_{o d}+v_{i n}\right) / L E_{\text {sat }}+1}{\left(\left(V_{o d}+v_{i n}\right) / L E_{\text {sat }}+1\right)^{2}} \mathrm{~d} v_{i n} .
\end{aligned}
$$

Substituting (17) into (21) and completing the integration produces

$$
A^{2}=V_{o d}^{2} \frac{(\rho+1)^{2}(\rho+2)\left(1-G_{c}\right)}{\rho\left(\rho(\rho+2)\left(1-G_{c}\right)+1\right)}=V_{o d}^{2} \mathcal{R}
$$

where $A \leq V_{o d}$ is desirable to avoid subthreshold onset (hardclipping). Then, from (22), $\mathcal{R} \leq 1$ and $1-\mathrm{dB}$ compression (i.e.,
$\left.G_{c}=0.9\right)$ constrain $0.5 \leq \rho \leq 2$. Within this range, the maximum $\mathcal{R}=1$ when $\rho=0.5$ or 2 , thereby allowing for the maximum $v_{\text {sig }}$ be applied to the input of the LNA. $A$ in (22), which corresponds to power $P_{\text {comp }}=A^{2} / 2 Z_{0}$, is the signal amplitude, resulting in the $G_{c}$-level of compression. From (22) it is possible to obtain

$$
P_{\text {comp }}=V_{\text {od }}^{2} \frac{\mathcal{R}}{2 Z_{0}} \text {. }
$$

Note that, if $G_{c}=0.9$, then $P_{\text {comp }}=I P_{1 d B}$. Furthermore, by using (23) and (19) in (12) and combining the constants on the right-hand side (labeled as $\mathcal{L}$ ), a relationship between linearity, power consumption, transistor length, and frequency can be expressed as

$$
\frac{P_{\text {comp }}}{P_{d} L f_{0}}=\frac{4 \pi}{v_{\text {sat }}} \frac{\sqrt{\left(1-|c|^{2}\right)}}{|c|} \frac{(\rho+1) \mathcal{R}}{3 \rho}=\mathcal{L} \frac{(\rho+1) \mathcal{R}}{3 \rho} .
$$

Equation (24) reaches $\mathcal{L}$ when $\rho=0.5$ and $0.5 \mathcal{L}$ when $\rho=2$. Therefore, $\rho=0.5$ will be used in Section IV-B to maximize (24).

The derivations of (19) make assumptions that are consistent with the derivations of (20) and Assumption \#5 (i.e. minimizing $V_{d s}=V_{d d}$ to $V_{d d}=V_{o d}$ ). While such low $V_{d s}$ may lead to clipping, since $V_{d d}$ clipping is not a prominent cause for gain compression (see the regression-analysis discussion above), no unduly clipping due to $V_{d d}$ is considered when deriving (24). The impact of $V_{d d}=V_{o d}$ is in increasing $\mathcal{L}$, which will form a limit on FoM in Section IV-B.

\section{Figures OF MERIT}

Based on the expressions in Section III, four FoMs are suggested.

\section{A. Noise and Sensitivity FoMs}

Having captured key low-noise design trade-offs in (20), the noise FoM is proposed as ${ }^{2}$

$$
F_{0} M_{N} \equiv \frac{F_{M} P_{d}^{1 / 3}}{L^{4 / 3} f_{0}^{2 / 3}}
$$

where $L$ is in $\mu \mathrm{m}, f_{0}$ is in $\mathrm{GHz}$, and $P_{d}$ is in $\mathrm{mW}$. $F_{M}$ from (8) replaces $\left(F_{\min }-1\right)$ in (20) to form (25) as motivated in Section II-B. Note that $F_{M}$ depends on gain via (8). Since most papers do not specify available gain, $F_{M}$ in (25) would be calculated based on the gain specified. For LNAs that achieve their $F_{\text {min }}$ and have large gain, $F_{M}$ reduces to $F_{m i n}-1$, and, as defined in (20), FoM $M_{N}$ comes close to achieving its limit, which is found via

$$
F_{o M_{N, \text { limit }}}=\frac{\left(10^{-6} \mathrm{~m}\right)^{4 / 3}\left(10^{9} \mathrm{~Hz}\right)^{2 / 3}}{\left(10^{-3} \mathrm{~W}\right)^{1 / 3}} \mathcal{N}\left(\alpha_{\text {opt }}, \rho_{\text {opt }}\right) \approx 0.4,
$$

where the terms preceding $\mathcal{N}$ account for the units of parameters used in (25), $\mu_{\text {eff }}=2 v_{\text {sat }} / E_{\text {sat }}, v_{\text {sat }}=8.43 \times 10^{4} \mathrm{~m} / \mathrm{s}, E_{\text {sat }}=4.7 \times$ $10^{6} \mathrm{~V} / \mathrm{m}$. Since $\rho$ impacts $\mathcal{N}$ in $(20)$ and since $\left|S_{11}\right|<-10 \mathrm{~dB}$, rather than the perfect power match, is generally acceptable, LNAs biased with $\rho<\rho_{\text {opt }}$ may realize $F o M_{N}$ that are closer to $F_{o M_{N, l i m i t}}$ than comparable LNAs with $\rho=\rho_{\text {opt }}$. This potential $F o M_{N}$ lowering, if any, is relatively small and comparison to $F_{o M_{N, l i m i t}}$ remains meaningful.

Sensitivity identifies the minimum detectable signal for a given noise factor. Thus, the sensitivity FoM can be defined based on $F o M_{N}$ as

$$
F_{o} M_{S} \equiv \frac{L^{4 / 3} f_{0}^{2 / 3}}{F_{M}}\left(\frac{P_{d}}{\Delta f}\right)^{-1 / 3}
$$

2 "Noise FoM" is the short-form name of a noise-gain-power-frequencytechnology FoM that models design trade-offs between these parameters and, akin to LNA noise factor, is expected to be reduced during an LNA design. 

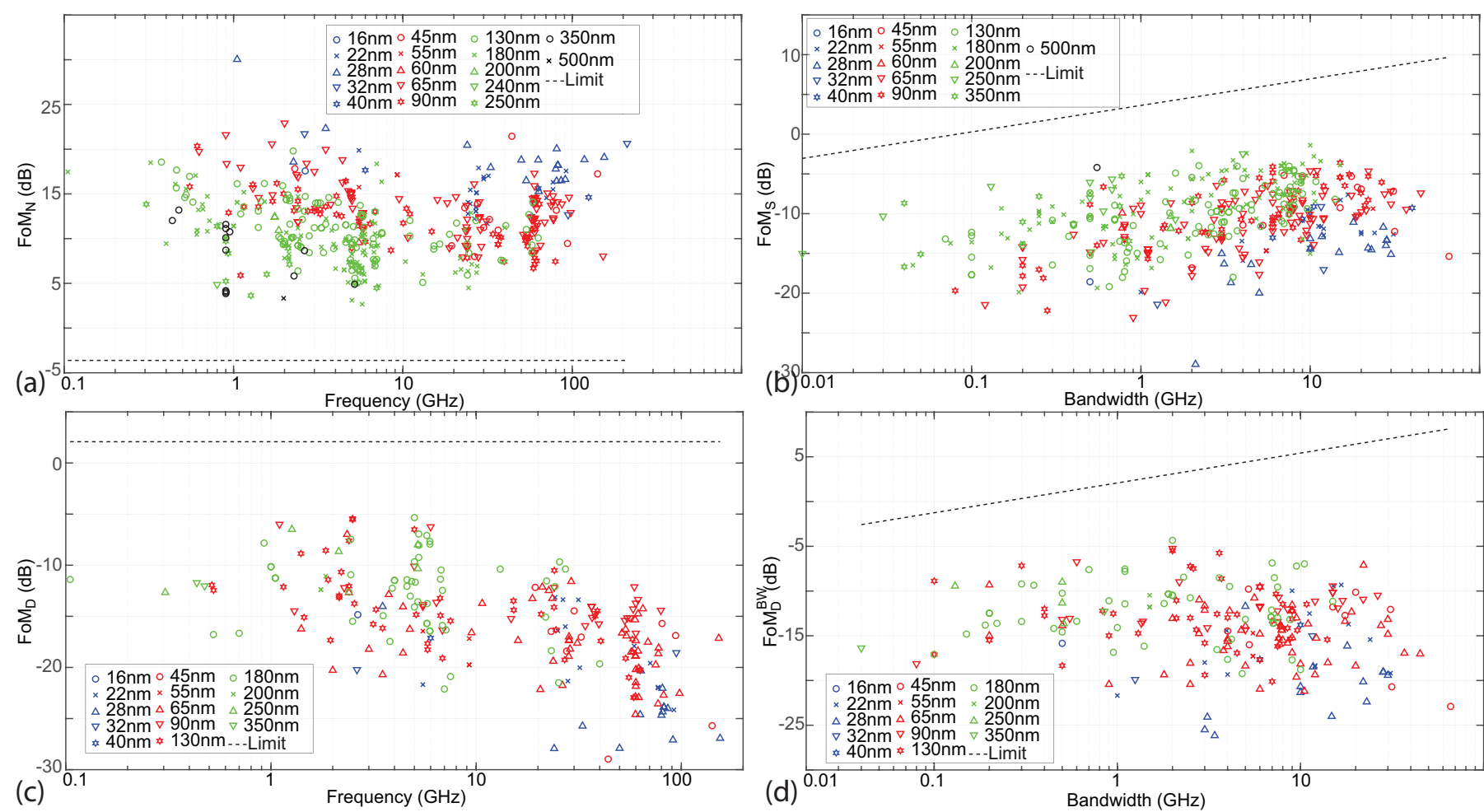

Fig. 1. Comparison of FoMs calculated for CMOS LNAs based on the data provided in [6], along with their limit lines.

where bandwidth $\Delta f$ is introduced to normalize $P_{d}$ to that of a narrowband LNA and is defined based on either $S_{11}$ or gain as appropriate for a given application. After introducing the relative bandwidth $B_{r} \equiv \Delta f / f_{0},(27)$ is rewritten as

$$
F o M_{S}=\frac{f_{0}^{1 / 3} B_{r}^{1 / 3}}{F o M_{N}}=\frac{L^{4 / 3} f_{0} B_{r}^{1 / 3}}{F_{M} P_{d}^{1 / 3}} .
$$

The limit to $\mathrm{FoM}_{S}$

$$
\text { FoM } M_{S, \text { limit }} \approx 2.5 f_{0}^{1 / 3} B_{r}^{1 / 3}
$$

depends on $1 / F_{o} M_{N, \text { limit }}$, the operating frequency, and bandwidth, where the maximum $B_{r}=2$.

\section{B. Dynamic-Range FoMs}

An intermediate FoM (i.e. a linearity FoM) can be based on (24):

$$
F o M_{L}=\sqrt[3]{\frac{P_{L}}{P_{d} L f_{0}}}
$$

In (30), $P_{\text {comp }}$ is replaced with the linearity measure, $P_{L}$ in $\mathrm{mW}$, as defined in $(11)$ with input-referred $\mathrm{P}_{1 \mathrm{~dB}}\left(I P_{1 \mathrm{~dB}}\right)$. Furthermore, the cubic root of the left-hand side of (24) is then taken to give (30). This cubic root is introduced intentionally to obtain $P_{d}^{1 / 3}$ (as in $F o M_{N}$ ), and does not alter the interdependence of its constituent parameters. Consequently, the trade-offs between all parameters in $F_{o} M_{N}, F_{o} M_{S}$, and $F_{o} M_{L}$ are relative to $P_{d}^{1 / 3}$, thereby permitting the relative comparison of these FoMs. The limit for $F_{o M}$ can also be obtained using $\mathcal{L}^{1 / 3}$ from (24). On its own, this limit is not very useful because the linearity of a single transistor, which led to (24), can be improved by adding additional transistors, and many LNAs would be expected to outperform this "limit." However, while additional transistors can improve linearity, they also increase noise measure and worsen $F o M_{N}$. Therefore, a dynamic-range FoM, which relates $F o M_{L}$ and $F o M_{N}$, is proposed as

$$
F_{o M_{D}} \equiv \frac{\text { FoM }_{L}}{\text { FoM }_{N}}
$$

Substituting (25) into (31) gives

$$
F_{O} M_{D}=\frac{P_{L}^{1 / 3} L f_{0}^{1 / 3}}{F_{M} P_{d}^{2 / 3}} .
$$

For a 1 -dB compression, where $G_{c} \approx 0.9$ and $\rho=0.5$, the limit on $F o M_{D}$ is found by substituting the values of the constants in the right-hand side of (24) to obtain

$$
\text { FoM }_{D, \text { limit }} \approx \frac{\left(10^{-3} \mathrm{~W}\right)^{1 / 3}}{\left(10^{-6} \mathrm{~m}\right) \times\left(10^{9} \mathrm{~Hz}\right)^{1 / 3}} \frac{\mathcal{L}^{1 / 3}}{\mathcal{N}} \approx 1.6 .
$$

Note that $F_{o M}$ is not a substitute for a dynamic range $D R \equiv$ $I P_{1 d B} / F$. The two metrics have different purpose. $F o M_{D}$ identifies the interdependence of LNA performance parameters and prescribes the expected trade-offs between them. In contrast $D R$ shows the range between the smallest and largest useful signal levels.

Alternatively, when bandwidth is a consideration, FoM $M_{S}$ and $F_{o M_{L}}$ are used to define

$$
F o M_{D}^{B W}=F_{o} M_{L} \times F_{o} M_{S}=\frac{P_{L}^{1 / 3} L f_{0}^{2 / 3}}{F_{M} P_{d}^{2 / 3}} B_{r}^{1 / 3} .
$$

which has a bandwidth- and frequency-dependent limit of

$$
F_{o M_{D, \text { imit }}}^{B W} \approx 1.6 \times f_{0}^{1 / 3} B_{r}^{1 / 3}
$$

\section{DiscusSiON}

Using the data in the LNA survey [6], the four proposed FoMs are calculated and plotted in Fig. 1 along with the limit lines. 
In [9], a regression analysis was performed to identify trends in the LNA data to propose FoMs. The FoMs in [9, (3)-(5)] are equivalent to $\left(F o M_{N}\right)^{-1}, F_{O} M_{S}$, and $F o M_{D}^{B W}$ and can be written as

$$
\left\{\begin{array}{c}
\widetilde{F o M_{N}}=\frac{T_{c a s}^{1.1} P_{d}^{0.32}}{L\left(f_{0}+14.2\right)^{2}} \\
\widetilde{F o M_{S}}=\frac{L \times B_{r}^{0.32}\left(f_{0}+17.4\right)^{2.3}}{T_{\text {cas }} P_{d}^{0.31}} \\
\widetilde{F O M_{D}^{B} W}=\frac{O I P 3 \times L \times B_{r}^{0.32}\left(f_{0}+17.4\right)^{2.3}}{T_{c a s} P_{d}^{0.31}},
\end{array}\right.
$$

where $T_{\text {cas }}=T_{0} F_{M}$ and the $\sim$ indicates that these are empirical results based on a regression analysis, rather than the derived equations reported in this paper. These FoMs are based on LNA measurements that have been compiled over the last 20 years. The shape and the powers of the terms in (36) closely resemble the configurations of the FoMs proposed in Section IV. The most obvious exception is the frequency term, which has a power of $2 / 3$ in (25) and (34), 1 in (28), and $1 / 3$ in (32), in contrast, $14.2 \mathrm{GHz}$ and $17.4 \mathrm{GHz}$ offsets are added to $f_{0}$ in (36), and the powers of 2 and 2.3 are used. These offsets allowed the regression analysis to provide a significantly better fit to the data and enabled better modeling of the measured noise factors that exhibit changes in slope with frequencies near these offsets. The change in slope follows the existing data rather than the theoretical expectations that form the basis of (25), (28), (32), and (34). However, the best least-squares power fit to $\left(f_{0}+14.2\right)^{2}$ and $\left(f_{0}+17.4\right)^{2.3}$ from 0.1 to $10 \mathrm{GHz}$ takes the form of $f_{0}^{0.3}$ and $f_{0}$ with $R^{2}$ values of 0.84 and 0.99 , respectively. This fit reflects the theoretical expectations in the FoMs proposed in this work albeit with a larger difference (2/3 vs 1$)$ for the power of $f_{0}$ in $F o M_{D}^{B W}$ and $\widetilde{F o M_{D}^{B} W}$. When deriving $\widetilde{F o M_{D}^{B} W}$, [9] did not identify much correlation between the output-referred IP3 (OIP3) and the other parameters in the data, and, as a result, $\widetilde{F O M_{S}}$ was simply multiplied by OIP3 to realize $F o M_{D}^{B W}$. Also, unlike Assumption \#2, $C_{g s}$ is not the only parasitic capacitance present in real circuits; indeed, the frequency dependence is expected to become more pronounced at high frequencies as reflected in (36). In addition, the power term for $L$ is slightly lower in (36) than in (25) and (28). The data shows that the power term for $L$ is lower than 1 for LNAs with $L>0.65 \mathrm{~nm}$, and higher than 1 when $L \leq 65 \mathrm{~nm}$. For $F_{o} M_{D}^{B W}$, the power of $L$ is the same as in $\widehat{F o M_{D}^{B} W}$. Fig. 1(c) shows increased gap between $F o M_{D}$ and $F o M_{D, \text { limit }}$ above $10 \mathrm{GHz}$, possibly due to more pronounced higher-order reactive distortions.

In this work, $\mathrm{P}_{1 \mathrm{~dB}}$ was selected as a linearity parameter to be used in $F o M_{D}^{B W}$. The relationships between $\mathrm{P}_{1 \mathrm{~dB}}$ and $L, P_{d}$, and $f_{0}$ were identified given the LNA-related assumptions. An additional $\sim 70$ LNAs have been added to [6] since the publication of [9]. The current trends in [6] indicate a reduction in the power of $P_{d}$ to 0.2 and a reduction in the power of $L$ to 0.9 .

\section{CONCLUSion}

This article identified LNA-related performance trade-offs based on the theoretical description of single-transistor circuits. Seven assumptions, made during these derivations, are clearly identified. From these trade-offs, four FoMs were proposed, and their limits with respect to LNA performance were derived. These FoMs normalize trade-offs by transistor length aiming to highlight the merits of LNA design methodologies. The FoMs only use data that are readily available in literature. Finally, the identified FoMs were compared to the results of a regression analysis of experimental results for nearly 500 CMOS LNAs reported over the past 20 years [6]. As the results of this comparison showed, the identified FoM exhibited reasonable agreement with the data in [6], even for the simplified model used in this work.

\section{REFERENCES}

[1] B. Murmann. ADC performance survey 1997-2021. [Online]. Available: http://web.stanford.edu/ murmann/adcsurvey.html

[2] H. Wang et al. Power amplifiers performance survey 2000-present. [Online]. Available: https://gems.ece.gatech.edu/PA_survey.html

[3] K. Makinwa. Smart temperature sensor survey. [Online]. Available: http://ei.ewi.tudelft.nl/docs/TSensor_survey.xls

[4] D. D. Wentzloff. Low power radio survey. [Online]. Available: www.eecs.umich.edu/wics/low_power_radio_survey.html

[5] J. Rimmelspacher. Integrated VCO performance survey. [Online] Available: https://www.elektronik.ovgu.de/Rimmelspacher_VCO

[6] L. Belostotski. Low-noise-amplifier (LNA) performance survey. [Online]. Available: https://schulich.ucalgary.ca/contacts/leo-belostotski

[7] X. Gao et al., "Jitter analysis and a benchmarking figure-of-merit for phase-locked loops," IEEE Transactions on Circuits and Systems II: Express Briefs, vol. 56, no. 2, pp. 117-121, 2009.

[8] W. Bae, "State-of-the-art circuit techniques for low-jitter phase-locked loops: Advanced performance benchmark FOM based on an extensive survey," in 2021 IEEE International Symposium on Circuits and Systems (ISCAS), 2021, pp. 1-5.

[9] L. Belostotski and S. Jagtap, "Down with noise: An introduction to a low-noise amplifier survey," IEEE Solid-State Circuits Magazine, vol. 12, no. 2, pp. 23-29, 2020.

[10] T. H. Lee, The Design of CMOS Radio-Frequency Integrated Circuits, 2nd, Ed. New York: Cambridge Univeristy Press, 2004.

[11] G. Banerjee, K. Soumyanath, and D. J. Allstot, "Desensitized CMOS low-noise amplifiers," IEEE Transactions on Circuits and Systems I: Regular Papers, vol. 55, no. 3, pp. 752-765, 2008.

[12] J.-S. Goo et al., "A noise optimization technique for integrated lownoise amplifiers," IEEE Journal of Solid-State Circuits, vol. 37, no. 8, pp. 994-1002, August 2002.

[13] L. Belostotski, "On the number of noise parameters for analyses of circuits with MOSFETs," IEEE Transactions on Microwave Theory and Techniques, vol. 59, no. 4, pp. 877-881, April 2011.

[14] J. Lerdworatawee and W. Namgoong, "Low-noise amplifier design for ultrawideband radio," IEEE Transactions on Circuits and Systems I: Regular Papers, vol. 51, no. 6, pp. 1075-1087, 2004.

[15] L. Belostotski and J. W. Haslett, "Two-port noise figure optimization of source-degenerated cascode CMOS LNAs," Analog Integrated Circuts and Signal Processing, vol. 55, no. 2, pp. 125-137, May 2008.

[16] D. K. Shaeffer and T. H. Lee, "A 1.5-V, 1.5-GHz CMOS low noise amplifier," IEEE Journal of Solid-State Circuits, vol. 32, no. 5, pp. 745759, May 1997.

[17] L. Belostotski and J. W. Haslett, "Noise figure optimization of inductively degenerated CMOS LNAs with integrated gate inductors," IEEE Transactions on Circuits and Systems I-Regular Papers, vol. 53, no. 7, pp. 1409-1422, July 2006.

[18] E. Zailer, L. Belostotski, and R. Plume, "Wideband LNA noise matching," IEEE Solid-State Circuits Letters, vol. 3, pp. 62-65, 2020.

[19] H. A. Haus and R. B. Adler, "Optimum noise performance of linear amplifiers," Proceedings of the IRE, vol. 46, no. 8, pp. 1517-1533, 1958.

[20] H. T. Friis, "Noise figures of radio receivers," Proceedings of the IRE, vol. 32, no. 7, pp. 419-422, July 1944.

[21] W. Sansen, "Distortion in elementary transistor circuits," IEEE Transactions on Circuits and Systems II: Analog and Digital Signal Processing, vol. 46, no. 3, pp. 315-325, 1999.

[22] E. Klumperink and B. Nauta, "Systematic comparison of HF CMOS transconductors," IEEE Transactions on Circuits and Systems II: Analog and Digital Signal Processing, vol. 50, no. 10, pp. 728-741, 2003.

[23] B. Razavi, RF Microelectronics. Upper Saddle River, NJ, USA: Prentice-Hall, Inc., 1998.

[24] H. Zhang and E. Sánchez-Sinencio, "Linearization techniques for CMOS low noise amplifiers: A tutorial," IEEE Transactions on Circuits and Systems I: Regular Papers, vol. 58, no. 1, pp. 22-36, 2011. 